\title{
Intracavity Electromagnetically Induced Transparency
}

\author{
Mikhail D. Lukin, Michael Fleischhauer ${ }^{1}$, Marlan O. Scully \\ Department of Physics, Texas A\&M University, College Station, TX 77843 and Max-Planck-Institut für Quantenoptik, \\ 85748 Garching, Germany \\ Vladimir L. Velichansky \\ Lebedev Institut of Physics, 53, Leninsky Prospect, Moscow, 117924 Russia
}

\begin{abstract}
The effect of intracavity Electromagnetically Induced Transparency on the properties of optical resonators and active laser devices is discussed theoretically. A pronounced frequency pulling and cavity linewidth narrowing are predicted. The effect can be used to substantially reduce classical and quantum phase noise of the beat-note of optical oscillators. Fundamental limits of this stabilization mechanism are discussed as well as its potential application to high-resolution spectroscopy.
\end{abstract}

Electromagnetically Induced Transparency (EIT) $)^{2}$ is a dense-media analog of "dark resonances" 3 which occur in three-level $\Lambda$ systems driven by coherent optical fields. In recent years a number of potential applications of EIT have been described. These include, in particular, enhancement of non-linear optical processes ${ }^{4}$, high-resolution spectroscopy and optical magnetometry ${ }^{5,6}$.

In the present Letter we describe theoretically the effect of an intracavity induced transparency. When a dense ensemble of coherently prepared $\Lambda$ atoms is placed inside an optical resonator, the resonator response is drastically modified resulting in a frequency pulling ${ }^{7}$ and a substantial narrowing of spectral features. This effect can be used for the frequency-difference stabilization of lasers ${ }^{8}$ or other two-mode light sources such as broadband parametric oscillators. Here intracavity EIT results in locking of the beat-note to the resonance frequency of a two-photon transition between metastable atomic levels and causes a substantial reduction of quantum and classical noise in the beat signal. Possible applications include sensitive intracavity spectroscopy, novel frequency standards and optical magnetometry.

The profound effects of intracavity EIT are due to the large dispersion close to the point of almost vanishing absorption $^{9}$ which can easily exceed the empty-cavity dispersion in the case of an optically thick $\Lambda$-medium. To illustrate the locking and narrowing mechanism let us consider a ring cavity containing a cell of length $l$ with a linear dispersive medium. The medium response is characterized by the real $\left(\chi^{\prime}\right)$ and imaginary part $\left(\chi^{\prime \prime}\right)$ of the susceptibility, for which we assume $\chi^{\prime}=\beta\left(\nu-\nu_{0}\right)$ and constant $\chi^{\prime \prime}$ for frequencies $\nu$ sufficiently close to some resonance frequency $\nu_{0} . \beta$ and $\chi^{\prime \prime}$ are proportional to the atomic density $N / V$. The cavity response function, i.e. the ratio of circulating to input intensity is given by ${ }^{10}$

$$
S(\nu)=\frac{I_{\text {circ }}}{I_{\text {in }}}=\frac{t^{2}}{1+r^{2} \kappa^{2}-2 r \kappa \cos [\Phi(\nu)]}
$$

where $t$ and $r$ are transmitivity and reflectivity of the input coupler $\left(t^{2}+r^{2}=1\right), \Phi(\nu)=\nu L / c+k l \chi^{\prime} / 2 \approx$ $\nu L / c+k l \beta\left(\nu-\nu_{0}\right) / 2$ is the total phase shift and $\kappa=\exp \left\{-k l \chi^{\prime \prime}\right\}$ describes the medium absorption per round trip $L$. Upon inspection of the round trip phase shift one finds that the resonance frequency of the combined cavity + medium system $\left(\Phi\left(\nu_{r}\right)=2 m \pi\right)$ is governed by a pulling equation:

$$
\nu_{r}=\frac{1}{1+\eta} \nu_{c}+\frac{\eta}{1+\eta} \nu_{0} .
$$

Here $\eta=(c k / 2)(l / L) \beta$ defines a frequency-locking or stabilization coefficient and $\nu_{c}$ is the resonance frequency of the empty cavity. Similarly by expanding the cosine in Eq.(1) around $\nu_{r}$ one finds that also the width of cavity resonances $\Delta \nu$ is changed by the intracavity medium:

$$
\Delta \nu=C \frac{1-r \kappa}{\sqrt{\kappa}(1-r)} \frac{1}{1+\eta},
$$

where $C$ is the empty cavity linewidth. The first factor describes an enhancement of the effective cavity + medium width due to additional losses, while the second one describes the reduction due to the linear dispersion. In the case 
when EIT is established in an intracavity medium, the absorption can be negligible $\left(\chi^{\prime \prime} \rightarrow 0\right)$ whereas the dispersion is large resulting in a substantial line narrowing.

To quantify this conclusion we consider the response of the typical $\Lambda$ system (Fig.1a) driven by a strong laser field of Rabi-frequency $\Omega_{2}$ to the weak test field $\Omega_{1}$. The corresponding linear susceptibility near the two-photon resonance $^{11}$ is:

$$
\chi^{\prime}=\xi \frac{\gamma_{1}\left(\nu-\nu_{0}\right)}{\Omega_{2}^{2}}, \quad \chi^{\prime \prime}=\xi \frac{\gamma_{1} \gamma_{0}}{\Omega_{2}^{2}} .
$$

Here $\xi=\left(3 / 4 \pi^{2}\right)\left(N \lambda^{3} / V\right), \nu_{0}=\nu_{2}-\omega_{b_{1} b_{2}}, \nu_{2}$ is the drive frequency, and $\omega_{b_{1} b_{2}}$ the frequency of the $b_{1} \rightarrow b_{2}$ transition. In a situation typical for EIT, i.e. when the lower levels are metastable, $\gamma_{0}$ can be very small and thus the absorption can be made small $(\kappa \approx 1)$ even for a large density-length product in the atomic vapor cell. Under these conditions, the phase shifts are large even for a very small detuning, resulting in a large stabilization coefficient. The ultimate limit of stabilization can be obtained by imposing the condition that the residual absorption losses in the cell should not exceed the empty-cavity losses. One finds for the maximum stabilization coefficient

$$
\eta \leq \frac{C}{2 \gamma_{0}}
$$

We note that for a long-lived ground-state coherence the ratio $C / \gamma_{0}$ can become very large. In this case the effective resonance frequency of the cavity coincides with $\nu_{2}-\omega_{b_{1} b_{2}}$ and the cavity width can be reduced by several orders of magnitude whereas the photon losses are practically unaffected. The above conclusion is illustrated in Fig. $1 \mathrm{~b}$, where the cavity transmission function is shown for different atomic densities.

It is instructive to estimate the lower limit to the cavity linewidth. For a good cavity and the maximum stabilization coefficient as per Eq.(5) we find $\Delta \nu \rightarrow 4 \gamma_{0}$, i.e. a linewidth which can be orders of magnitude smaller than both empty-cavity linewidth and single-atom transparency window (width of $\Lambda$-resonance). In the strong field limit the latter is power broadened and scales like $\Omega_{2}$. Hence the effect of power-broadening on the combined cavity+atom system is here completely eliminated in the high-density regime.

Let us now discuss the effect of the $\Lambda$-medium on the phase-difference noise of two optical modes independently oscillating inside the cavity. A 3-level intracavity medium displaying EIT can be used to lock the beat-note to the resonance frequency of the two-photon transition $\omega_{b_{1} b_{2}}$. In particular, we will focus here on the spectral properties of a two-mode laser. We emphasize, however, that the two-mode laser serves only as a generic example. Alternatively, one can consider locking the beat-note of two independent single-mode lasers or of a broadband non-degenerate parametric oscillator. The two-mode lasers considered here are especially convenient when frequency differences are to be measured since the beat-note of the two modes can be intrinsically narrow provided that the optical paths are similar. The evolution of the coherent amplitudes $a_{1}$ and $a_{2}$ of the oscillating (laser) fields can be described by stochastic c-number equations $(n=1,2)$

$$
\dot{a}_{n}=-\left(\frac{C}{2}+i \Delta_{n}^{c}\right) a_{n}+\frac{A_{n}}{2} a_{n}+i g_{n} N \sigma_{n}+F_{n}(t)
$$

Here $A_{1,2}$ are the effective gain coefficients for the two modes, which have the generic structure $A_{1,2}=\alpha_{1,2}-$ $\beta_{1,2}\left|a_{1,2}\right|^{2}-\tilde{\beta}_{1,2}\left|a_{2,1}\right|^{2}$. The linear gain coefficients $\alpha_{n}$ as well as the self-saturation and cross-saturation coefficients $\beta_{n}$ and $\tilde{\beta}_{n}$ respectively depend on the specific laser model ${ }^{10}$. Their exact form is unimportant for the present discussion as long as $\beta_{1} \tilde{\beta}_{2}<\beta_{1} \beta_{2}$. $F_{n}$ are noise operators associated with the gain processes. Their correlation function is given by ${ }^{10}:\left\langle F_{n}(t)^{*} F_{n}\left(t^{\prime}\right)\right\rangle=C \delta\left(t-t^{\prime}\right) . g_{1,2}$ describe the coupling to the $\Lambda$-medium. $\Delta_{1,2}^{c}=\nu_{1,2}^{c}-\nu_{1,2}$; $\nu_{1,2}$ being the actual lasing frequencies of the two modes and $\nu_{1,2}^{c}$ are the corresponding eigenfrequencies of the empty cavity. Absorption, dispersion and noise properties of the $\Lambda$ atomic system are also described by a set of c-number Langevin equations for the polarizations $\sigma_{i}{ }^{11}$. In the following we restrict ourselves to a symmetric configuration and assume equal gain, cavity losses, coupling constants etc.

The semiclassical behaviour of the laser modes can be studied by disregarding all noise contributions and eliminating the atomic variables. The laser equations (6) have a solution with equal amplitudes of the modes $a_{1}=a_{2}$. In this case of equal strength of both fields we have close to the resonance: $\chi_{1,2}^{\prime}=\xi \gamma\left(\Delta_{2,1}-\Delta_{1,2}\right) /\left(2 \Omega^{2}\right), \chi_{1,2}^{\prime \prime}=\xi \gamma \gamma_{0} /\left(2 \Omega^{2}\right)$, with $\gamma=\gamma_{1}=\gamma_{2}, \Omega=g a_{1}=g a_{2}$ and $\Delta_{1,2}=\omega_{a b_{1,2}}-\nu_{1,2}$.

It is convenient to write the field equations in terms of square amplitudes (photon numbers) $n_{1,2}$ and phases $\phi_{1,2}$. The steady-state solution of the laser phase equations immediately leads to the frequency pulling equation: 


$$
\nu_{1}-\nu_{2}=\frac{1}{1+\bar{\eta}}\left(\nu_{1}^{c}-\nu_{2}^{c}\right)+\frac{\bar{\eta}}{1+\bar{\eta}} \omega_{b_{2} b_{1}}
$$

with $\bar{\eta}=\eta / 2$. For $\eta \gg 1$ the beat-note frequency is locked to $\omega_{b_{1} b_{2}}$, i.e. to the $\Lambda$-resonance frequency. The additional absorption in the cavity increases the effective decay rate for the laser modes by $\bar{\eta} \gamma_{0}$. This absorption is however unimportant provided that inequality (5) is fulfilled.

Let us now turn to the phase-noise properties of the two-mode laser. The noise contribution due to the interaction with the $\Lambda$-medium is negligible ${ }^{11}$. We model the effect of technical noise by a fluctuation of the spacing between the cavity resonance frequencies $\delta \omega_{c}=\omega_{1}^{c}-\omega_{2}^{c}$. The last is assumed to obey a linear stochastic equation with a Markovian noise force and a phenomenological damping rate $\gamma_{c}, \delta \dot{\omega}_{c}=-\gamma_{c} \delta \omega_{c}+F_{c}$ with $\left\langle F_{c}(t) F_{c}\left(t^{\prime}\right)\right\rangle=\gamma_{c}\left\langle\delta \omega_{c}^{2}\right\rangle \delta\left(t-t^{\prime}\right)$, where $\left\langle\delta \omega_{c}^{2}\right\rangle$ characterizes the strength of technical fluctuations. The stochastic equations can be solved by linearization and the beat-note phase-noise spectrum can be calculated. In the low (fluctuation-) frequency regime $(\omega \ll \Omega)$ we find:

$$
S(\omega)=\frac{1}{(1+\bar{\eta})^{2}} \frac{2 \gamma_{c}}{\omega^{2}+\gamma_{c}^{2}}\left\langle\delta \omega_{c}^{2}\right\rangle+\frac{2 C^{2}}{(1+\bar{\eta})^{2}} \frac{\hbar \nu}{P_{\text {out }}} .
$$

The two terms in Eq.(8) represent the influence of technical fluctuations and the beat-note phase diffusion of the laser (Shawlow-Townes linewidth ${ }^{10}$ ) respectively. Thus, due to the intracavity medium the (Markovian) technical as well as quantum fluctuations are reduced by a factor $1 /(1+\bar{\eta})^{2}$. Slow technical fluctuations, such as temperature drifts of the cavity resonances are reduced only by $1 /(1+\bar{\eta}$ ) (see Eq. $(7))$. Note that for strong stabilization $\left(\bar{\eta}=C / \gamma_{0} \ll 1\right)$ the phase-diffusion is proportional to $\gamma_{0}^{2}$ instead of $C^{2}$. Such a suppression of quantum phase noise in the laser beatnote $^{12}$ is a consequence of the cavity line narrowing effect described above.

We note here that in addition to the increased intracavity losses there exists another important limitation on the maximal $\eta$ value. It is due to the dynamical instabilities which often arise in different stabilization schemes ${ }^{13}$. For the present system the frequency pulling regime described above is stable as long as $\bar{\eta}<2 \gamma / \gamma_{0}$ for a homogeneously broadened system and $\bar{\eta}<2 \Delta_{D} / \gamma_{0}$ for a medium which is Doppler broadened (one photon Doppler width $\Delta_{D}$ ).

It is interesting to consider a particular example of the beat-note laser stabilization. A variety of the gas and dye lasers as well as certain types of extended cavity diode lasers can operate on two modes (possibly of orthogonal polarizations) with frequency separation on the order of a few GHz. In this case the frequency difference of the modes can be locked to the transition between hyperfine components of alkali atomic vapors. The natural linewidth of such two-photon transitions can be made as low as $10-100 \mathrm{~Hz}$ using buffer-gas or wall-coating techniques. Taking the empty cavity width $\sim 10^{7} \mathrm{~Hz}$ we find that atomic densities corresponding to the stabilization factor $\eta>10^{5}$ can be used without affecting the output power of the laser. This can be achieved in an alkali-vapor cell using transitions of the $\mathrm{D}$ absorption lines at moderate atomic densities $\sim 10^{12} \mathrm{~cm}^{-3}$ and laser intensities above optical saturation. Depending upon the initial degree of technical-noise correlation the resulting beat-note linewidths can be in or below the $\mathrm{mHz}$ region.

The potentially interesting feature of the present approach is that it allows one to combine strong locking of two laser modes and narrow linewidths with intense laser fields. It has already been demonstrated ${ }^{6}$ that dispersive effects in a dense coherent medium can be used to significantly reduce power broadening of two-photon resonances and thus can lead to a potentially attractive regime of laser spectroscopy where narrow resonances coexist with strong fields. An interferometric measurement in a dispersive medium typically leads to several narrow interferometric fringes. In practice it is therefore often difficult to distinguish and determine the position of the central fringe. This is no longer a problem if intracavity EIT is used, since in the regime of strong frequency pulling the beat-note automatically locks to the two-photon resonance, whereas the effective width is equivalent to the width of the interferometric fringes. These features make various applications of the presently proposed technique for improvement of atomic frequency standards and optical magnetometers feasible.

The authors gratefully acknowledge the support from the Office of Naval Research, the Welch Foundation, and the Texas Advanced Research and Technology Program. M. F. would like to thank the Alexander-von-Humboldt foundation for financial support. 
1. also: Sektion Physik, Ludwig-Maximilians-Universität München, Theresienstraße 37, D-80333 München, Germany

2. K.J. Boller, A . Imamo u glu, and S.E. Harris, Phys.Rev.Lett. 66, 2593 (1991); for review of the subject see S. E. Harris, Physics Today 50, \# 7, 36 (1997).

3. E. Arimondo, Progress in Optics XXXV, 259-354 (1996).

4. M. Jain, Hui Xia, G. Y. Yin, A. J. Merriam, and S. E. Harris, Phys. Rev. Lett., 77, 4326 (1996).

5. M. O. Scully and M. Fleischhauer, Phys. Rev. Lett. 69, 1360 (1992).

6. M. D. Lukin, M. Fleischhauer, A. S. Zibrov, H. G. Robinson, V. L. Velichansky, L. Hollberg, and M. O. Scully, Phys. Rev. Lett. 79, 2959 (1997).

7. Frequency pulling by "dark resonances" in an optically thin medium was discussed in A.M.Akulshin, A.A.Celikov, V.L.Velichansky, Optics Comm. 84, 139, (1991); A.M.Akulshin and M.Ohtsu, Quantum Electronics 24, 561 (1994).

8. We note here that the present system is related to the Correlated Emission Laser; M.P.Winters, J.L.Hall, and P.Toschek, Phys.Rev.Lett. 65, 3116 (1990), where a coherently prepared 3-level gain medium leads to a strong correlation of the phase fluctuations of two laser modes. The CEL effect results in a vanishing diffusion coefficient for the relative phase angle. In contrast to the present case it is however accompanied by phase locking.

9. S. E. Harris, J. E. Field, and A. Kasapi, Phys. Rev. A 46, R29 (1992); M. Xiao, Yong-qing Li, Shao-Zheng Jin, and J. Gea-Banacloche, Phys. Rev. Lett. 74, 666 (1995).

10. A. E. Siegman, Lasers, (University Science Books, Mill Valley, California, 1986); M. Sargent, M.O. Scully and W. Lamb, Laser Theory, (Addison-Wesley, Reading, Massachusets, 1974)

11. For a c-number Langevin description of $\Lambda$-type atoms interacting with two fields, see for example: M. Fleischhauer and Th. Richter, Phys. Rev. A 51, 2430 (1995).

12. An example of the use of dispersive elements for quantum noise reductions is described in A. Yariv, R. Nabiev, and K. Vahala, Opt. Lett. 15, 1359 (1990); Y.Shevy, J. Iannelli, J. Kitching, and A. Yariv, Opt.Lett. 17, 661 (1992).

13. H.Li and N.B.Abraham, Appl.Phys.Lett. 53, 2257 (1988).

14. O.Kocharovskaya and I.V.Koryukin, in Non-linear Dynamics in Optical Systems, vol.7, Eds. N.B.Abraham, E.Carmine and P.Mandel (OSA, Washington, DC 1991) p.251
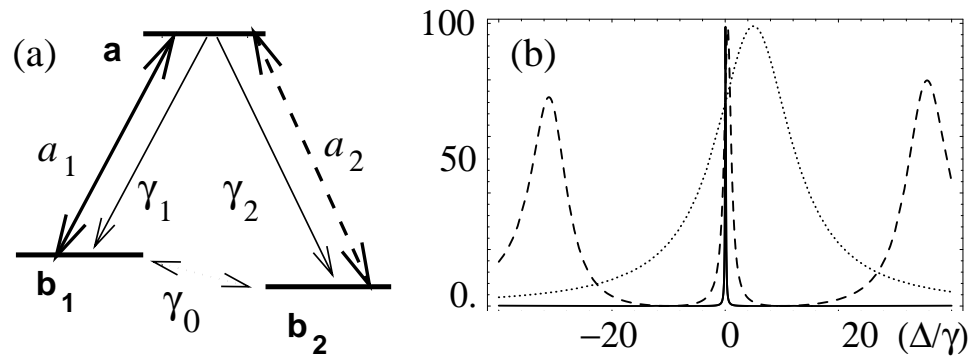

Fig.1

Fig. 1. a) A generic $\Lambda$-system for EIT. Frequencies of two fields are close to the resonant frequencies of transitions $a \rightarrow b_{1}$ and $a \rightarrow b_{2}$ respectively. $b_{1,2}$ are metastable states. b) Cavity response as a function of test field frequency for different values of atomic density. Dotted, dashed, and solid curves correspond to $\eta=0,10,100$ respectively. Parameters are $\Omega_{2}=10 \gamma, \mathrm{r}=$ $0.98, \nu_{c}-\nu_{0}=5 \gamma$. 